\title{
Resonancia magnética cardíaca con perfusión stress: Utilidad clínica y relación con coronariografía convencional
}

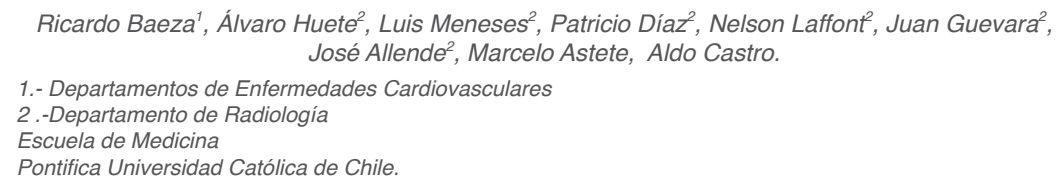

\section{Resumen:}

Introducción: La resonancia magnética cardiaca (RMC) es una herramienta no invasiva, libre de radiación que permite una evaluación global del corazón. Una de las potencialidades son los estudios de perfusión miocárdica con stress.

Objetivo: Presentar nuestra experiencia de RMC con stress (RMCS) su correlación con la coronariografía convencional (CC) y su relación con eventos clínicos.

Método: En forma prospectiva se incluyeron en un registro todos los pacientes sometidos a RMCS en nuestro servicio entre Enero 2007 y Abril 2009.

Todos los pacientes fueron sometidos a RMCS analizando anatomía, estructura, función global y segmentaria, perfusión stress/reposo y viabilidad miocárdica. Los exámenes fueron realizados en resonadores $1.5 \mathrm{~T}$, en apnea de \pm 10 segundos, con gatilleo ECG retrospectivo y con uso de Gadolinio endovenoso para las fases de perfusión y viabilidad El estudio de stress se realizó con inyección de adenosina ev (140ug/kg/min) tras lo cual se adquirieron las imágenes y comparadas con la fase de reposo. El estudio de viabilidad se realizo a continuación de la fase de reposo. Se consideraron como RMCS positivas aquellas con áreas de hipoperfusión en stress que se recuperaron en reposo. En aque- llos pacientes que fueron sometidos a CC dentro de los 3 meses de realizado la RMCS, se realizó correlación entre ambos métodos, considerando presencia y localización de la enfermedad coronaria. Se consideraron significativas las lesiones $\geq 70 \%$ de estenosis luminal.

Resultados: Incluimos 71 pacientes que fueron estudiados durante el periodo indicado. Las indicaciones para los exámenes fueron: estudio viabilidad e insuficiencia cardiaca (64\%); sospecha de cardiopatía coronaria $(36 \%)$. Las características del grupo fueron: Hombres 72\%, Edad $49 \pm 7$ años; Diabetes 17\%, HTA $13 \%$, AFCC $15 \%$, TBQ $20 \%$.

La fracción de eyección promedio fue de $53 \pm 7 \%$. El tiempo de examen fue de $50 \pm 5$ minutos. No hubo reacciones adversas al Gadolinio, ni al uso de adenosina endovenosa.

En 31 pacientes (42\%) se realizo CC. En estos la RMCS fue positiva para isquemia en $100 \%$ de los casos de los pacientes con CC que presentaban estenosis coronaria $\geq 70 \%$. Con un $90 \%$ de certeza para la localización de isquemia correlacionada con la CC.

Conclusión: El uso de la RMCS es factible, seguro y confiable en pacientes con sospecha de enfermedad coronaria significativa. Sus valores de sensibilidad y especificidad la hacen una herramienta atractiva para uso clínico.

Correspondencia: Dr. Ricardo Baeza V. 


\section{Stress Perfusion Cardiac Magnetic Resonance Imaging: Clinical usefulness compared to conventional coronary angiography.}

Background: Cardiovascular magnetic resonance (CMR) is a non invasive and non ionizing imaging tool that allows a complete cardiovascular evaluation. One of its advantages is the ability to analyze myocardial perfusion with and without stress.

Aim: To show our experience using stress perfusion CMR (SPCMR), its correlation with conventional coronary angiography (CCA) and cardiovascular events. Methods: We retrospectively included in the analysis all patients referred to our institution to undergo a SPCMR since January 2007 to April 2009. In all patients we performed a complete evaluation including anatomic, left ventricular function, adenosine stress perfusion and viability study. All the studies were performed in a $1.5 \mathrm{~T}$ scanner and 10 seconds breath-hold. Stress perfusion was performed alter iv adenosine bolus (140 $\mathrm{ug} / \mathrm{Kg} / \mathrm{min}$ ). Eight minutes after the stress, the rest phase was performed. Viability analysis was done after the iv injection of gadolinium based contrast $(0.15 \mathrm{mMol} /$ $\mathrm{kg}$ ). A positive SPCMR was considered in those with mismatch areas between stress and rest phases. In those patients who underwent a CCA, we correlated the SP-

\section{Introducción:}

La enfermedad coronaria (EC) sigue siendo una entidad prevalente en la medicina actual ${ }^{1}$. Si consideramos la tendencia de las últimas décadas, la mayor expectativa de vida de los pacientes y además la prevalencia de los factores de riesgo convencional se puede presumir fácilmente que esta cifra irá en aumento en los próximos años.

La EC se manifiesta de diferentes formas en la práctica clínica, teniendo un espectro que va desde la muerte súbita, pasando por los cuadros de angina inestable y el infarto miocárdico con supradesnivel del segmento ST. Sin embargo, en la práctica clínica muchas veces el diagnóstico de EC no es fácilmente establecido y se requieren de herramientas diagnósticas complementarias. Estas últimas tienen por objetivo descartar o confirmar la presencia de enfermedad coronaria. Las técnicas diagnósticas más utilizadas van desde el simple ECG, la ergometría de esfuerzo, la ecografía con stress farmacológico y las técnicas de medicina nuclear con isótopos. Cada una de estas tienen
CMR findings with the presence and location of stenoses equal or greater than $70 \%$ as significant.

Results: We included 71 patients during the mentioned period. Indication for SPCMR were heart failure and viability study (64\%); coronary ischemia (36\%). Main baseline characteristics were: Male 7\%; age $9 \pm 7$ years; diabetes $17 \%$; hypertension $13 \%$; previous family history for CAD $15 \%$; and tobacco $20 \%$.

Mean left ventricular ejection fraction was $53 \pm 7 \%$. Mean time to complete the exam was $50 \pm 5$ minutes. There were not adverse reactions to contrast or adenosine.

Thirty one patients (42\%) underwent CCA. All patients with stenoses greater than $70 \%$ had a positive SPCMR. Agreement between CCA and SPCMR for ischemia location was $90 \%$.

Conclusion: SPCMR is feasible, safe and reliable in patients with suspected ischemia. Sensitivity, specificity and predictive values make this non invasive technique a very appealing tool in clinical practice.

Keywords: Cardiac Magnetic Resonance, coronary angiography

limitaciones y también distintos valores de sensibilidad y especificidad en las poblaciones estudiadas, siendo las técnicas de medicina nuclear la más sensibles y específicas hasta la fecha. En la última década una de las técnicas que se ha introducido en el campo de la cardiología es la resonancia magnética cardíaca (RMC), la cual dentro de sus capacidades tiene la posibilidad de evaluar la presencia de isquemia y enfermedad coronaria ${ }^{2}$.

La RMC es una técnica de imagen no invasiva, libre de radiaciones ionizantes y que es capaz de obtener imágenes de alta resolución del tejido cardíaco, lo cual permite evaluar aspectos tales como la morfología, función ventricular, masas y tumores, viabilidad e isquemia miocárdica. Para lograr esta última, se realiza una técnica llamada RMC con perfusión stress (RMCPS), la cual consiste en utilizar un stress farmacológico que permita evidenciar la diferencia en la captación del medio de contraste por el miocardio entre zonas normo e hipo-perfundidas. Este principio es el mismo utilizado en técnicas como la medicina nuclear y el cual está ampliamente validado. En 
la práctica clínica se utilizan dos tipos de stress farmacológico, los que producen cambios en la contractilidad, como la dobutamina, y los que producen un efecto de vasodilatación coronaria permitiendo objetivar el defecto de perfusión, como es el caso de la adenosina o el dipiridamol. Los protocolos para las distintas técnicas han sido publicados en la literatura, sin embargo, no existen hasta la fecha trabajos comparativos entre ellas. Desde el punto de vista clínico la RMC con perfusión stress (RMCPS) es una técnica atractiva dado que además agrega la información obtenida con el resto del examen (función, anatomía, contractilidad segmentaria) ${ }^{3,4}$.

El presente trabajo tiene por objetivo describir la experiencia clínica con la RMCPS y la correlación de ésta con un subgrupo que se sometió coronario-grafía convencional, en una población referida para RMCS.

\section{Método:}

Selección de Pacientes: Desde enero 2007 hasta abril 2009 , se incorporaron todos los pacientes que fueron referidos a nuestro centro para ser sometidos a RMCPS y que no presentaran contraindicación para ser sometidos al examen. Dentro de las contraindicaciones para ser sometidos a RMCPS se consideraron la presencia de marcapasos definitivos y/o desfibriladores, implantes cocleares, claustrofobia severa y función renal alterada (creatinina sérica mayor a $2 \mathrm{mg} / \mathrm{dL}$ ). Además, por el uso de adenosina se les solicitó a los pacientes no consumir xantinas y/o derivados el día previo al examen.

\section{Resonancia Magnética:}

a) Protocolo: El protocolo de RMCPS está esquematizado en la Figura 1. Todos los pacientes fueron estudiados en equipo de $1.5 \mathrm{~T}$ y en los cuales se efectuó el mismo protocolo de estudio. Tras obtener los localizadores, se obtuvieron imágenes de cine usando técnica de Steady state free presesion (SSFP), obteniendo un tamaño de voxel de 1.6x1.7x6 mm. Para el análisis de esta secuencia se obtuvieron imágenes en ejes cortos cubriendo desde la base hasta el ápex, y en ejes largos en modos de 2, 3 y 4 cámaras. Terminada la fase de cine, se procedió al protocolo de perfusión stress. Para este efecto los pacientes recibieron adenosina como fármaco vasodilatador en dosis de 140ug/ $\mathrm{Kg} / \mathrm{min}$, infundiéndola en 2 minutos. Inmediatamente terminada la adenosina se procedió con la secuencia para la obtención de imágenes de stress en eje corto. Se obtuvieron cortes a nivel de la base, zona media y ápex del ventrículo izquierdo, utilizando la imagen en cuatro cámaras para definir los segmentos a estudiar. En aquellos pacientes que presentaban alteración de la contractilidad segmentaria en la fase de cine, se definieron esos segmentos para el análisis. Durante la fase de stress, inmediatamente posterior a la inyección de adenosina, se administró Gadolinio $(0.075 \mathrm{mmol} / \mathrm{Kg})$ por una vía venosa exclusiva. Esta dosis de gadolinio fue repetida 8-10 minutos después

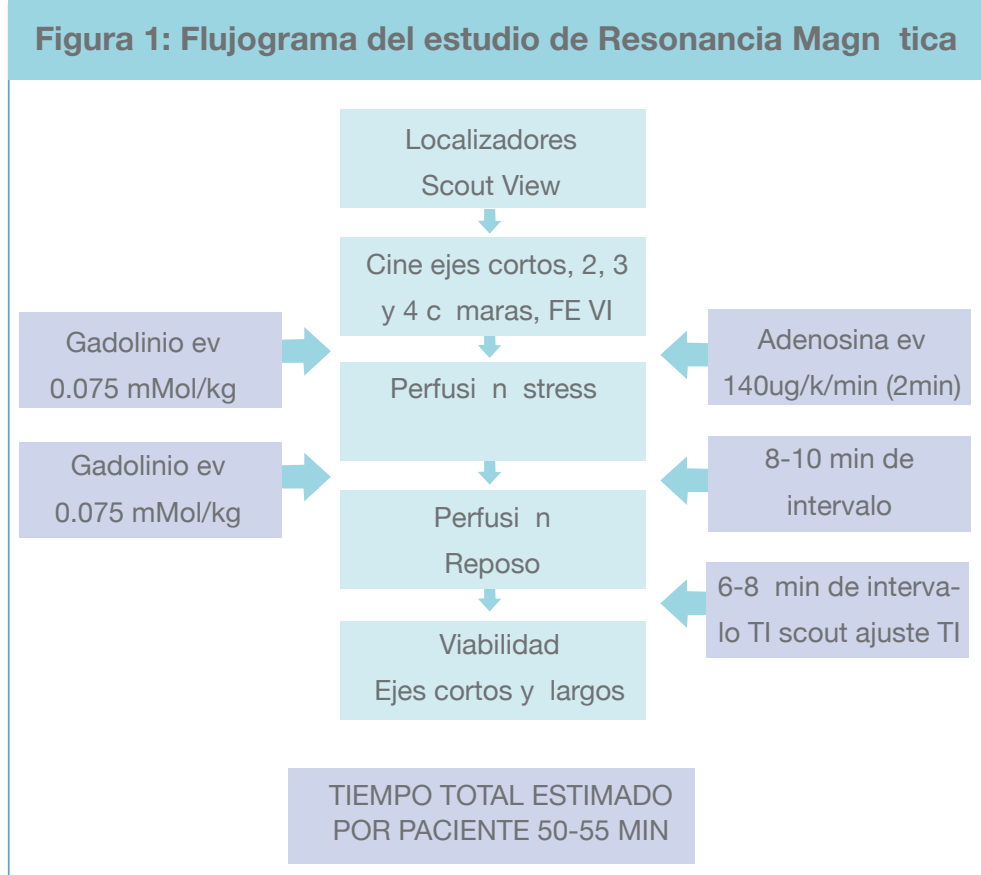

Figura 1. Flujograma del examen de Resonancia Magnética con perfusión stress. 
de terminada la fase de stress, para complementar la fase de reposo. Tanto la fase de stress como de reposo se hicieron con respiración libre. Tras la realización de la fase de reposo, se realizó la fase de viabilidad, seis a ocho minutos terminados la última inyección de Gadolinio. Para esta fase se ajustó un TI (tiempo de inversión) necesario para obtener un realce de las zonas de necrosis miocárdica. El estudio de viabilidad se hizo con técnica de TURBO FLASH y en apneas de 10 a 12 segundos.

b)Análisis de los Exámenes: Todas las RMCS fueron informadas siguiendo el mismo formato, inmediatamente tras la realización del examen. Aquellos pacientes que fueron estudiados con coronariografía convencional, la información de la RMCPS estuvo disponibles antes del examen. El análisis de función global y segmentaria fue realizado en forma independiente por un observador y se entregó una estimación visual de la fracción de eyección del ventrículo izquierdo (FE) y además de la función contráctil segmentaria. Esta última fue informada siguiendo el esquema de 17 segmentos que ha sido previamente publicado. Los análisis de las fases de perfusión fueron realizados considerando como zonas isquémicas aquellas áreas en las cuales no hubo captación del Gadolinio durante el stress y que se recuperó en la fase de reposo. Aquellas zonas que mostraron hipocaptación, tanto en las fases de stress como reposo, fueron consideradas como artefacto, salvo que estas zonas captaran Gadolinio en la fase de viabilidad, lo cual se consideró como necrosis miocárdica. Para el análisis de esta última fase se informaron con zonas de necrosis todas aquellas que captaron Gadolinio siguiendo un patrón isquémico (esto es desde el endocardio hacia el epicardio). Además se complementó el informe con la localización de la necrosis y la extensión de ella. No se realizó un cálculo cuantitativo de las zonas de necrosis. Coronariografía Convencional: Un grupo de los pacientes estudiados fueron referidos para coronariografía convencional (CC). La indicación de la CC fue basada en decisión clínica del médico tratante. Estas fueron realizadas por vía radial o femoral según decisión del intervencionista siguiendo el protocolo habitual. Se consideraron como significativas todas las lesiones coronarias que significarán un $70 \%$ o más de estenosis en un vaso epicárdico. El reporte de las lesiones coronarias fue independiente y por separado del reporte de la RMCPS.

Análisis Estadístico: Las variables categóricas fueron comparadas usando test de Chi cuadrado o test de Fisher. Para el análisis de certeza del test diagnóstico, presencia de enfermedad coronaria y localización, se utilizó test de Kappa. Las variables numéricas fueron comparadas con test de T o test no paramétrico (Mann-Whitney). Se consideró un error alfa $=0.05$ en todos los análisis.

\section{Resultados:}

Durante el período señalado se estudiaron 71 pacientes referidos para RMCPS de los cuales 31 fueron referidos para estudio invasivo. Las principales características demográficas de la población total y del subgrupo estudiado con coronariografía están descritas en la Tabla 1. No hubo diferencias significativas de las características entre ambos grupos estudiados.

La RMCPS fue positiva para isquemia en 12 (17\%) de los pacientes. Destaca un grupo con una buena función ventricular izquierda. De los 31 pacientes que fueron sometidos a CC el test fue positivo para isquemia en $38 \%$ de los casos. La fracción de eyección promedio para el grupo fue de $53 \pm 7 \%$. No se registraron reacciones adversas por

\section{Tabla 1: características demográficas de los pacientes sometidos a Resonancia Magnética Cardíaca con perfusión stress (RMCPS) y del subgrupo estudiado con coronariografía convencional.}

\begin{tabular}{|c|c|c|c|}
\hline & Población Total $\mathbf{N}=71$ & Con Coronariografía N=31 & $\mathbf{P}$ \\
\hline Edad (a os), DS & $48 \pm 7$ & $52 \pm 2$ & ns \\
\hline Hombres, n (\%) & $50(70)$ & $16(50)$ & ns \\
\hline HTA, n (\%) & $9(13)$ & $6(18)$ & ns \\
\hline Diab ticos, n (\%) & $12(17)$ & $8(24)$ & ns \\
\hline Fumadores, n (\%) & $14(20)$ & $6(17)$ & ns \\
\hline AFCC & $11(15)$ & $6(17)$ & ns \\
\hline IAM previo, n (\%) & $12(17)$ & $5(16)$ & ns \\
\hline AP previo, n (\%) & $12(17)$ & $5(16)$ & ns \\
\hline
\end{tabular}




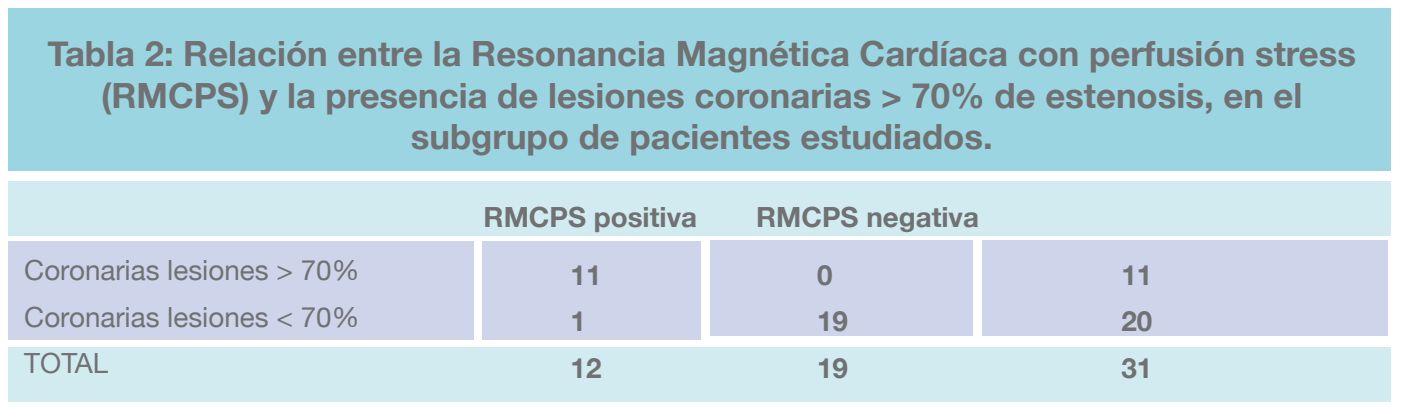

uso de Gadolinio ni por el uso de adenosina.

Desde el punto de vista clínico, no hubo reacciones adversas al medio de contraste ni tampoco complicaciones por el uso de adenosina. En el seguimiento aquellos pacientes que tuvieron RMCPS negativa para isquemia y no fueron estudiados con coronariografía, no se registraron eventos cardiovasculares adversos. Todos los pacientes que tuvieron una RMCPS positiva para isquemia fueron estudiados en forma invasiva.

La Tabla 2 muestra los hallazgos de la RMCPS en el subgrupo que fue estudiado con CC y la correlación entre ambas técnicas. Como se puede apreciar la RMCPS fue positiva en los 11 casos que tenían lesiones coronarias significativas (sensibilidad $=100 \%$ ), y fue negativa en 19 de los casos que no tenían EC significativa (especificidad $=95 \%)$. Destacan los coeficientes de kappa= 0.931 ( $\mathrm{p}<0.001)$ para la correlación entre el diagnóstico de enfermedad coronaria significativa y Kappa $=0.824$ para la concordancia entre ambas técnicas para localizar la zona de isquemia. Los valores predictivos, positivos y negativos, fueron $91 \%$ y $100 \%$ respectivamente en esta serie. Hubo una paciente en la cual la RMCPS fue positiva, pero en la que no se demostraron lesiones coronarias epicárdicas.

La Figura 2 muestra el caso de paciente con antecedentes de angioplastía en dos arterias coronarias y que fue hospitalizado por dolor torácico, pero sin cambios electrocardiográficos ni alza de enzimas cardíacas. Por decisión de su tratante se solicitó estudio de isquemia. Como muestran las imágenes, existe un defecto de perfusión en la cara lateral del ventrículo izquierdo durante la fase de stress, el cual revierte durante la fase de reposo y por lo tanto compatible con el diagnóstico de isquemia. El estudio coronariográfico realizado demostró re-estenosis de uno de los stents, específicamente en la arteria lateral alta, tras lo cual se procedió a una nueva angioplastía.

\section{Figura 2: Hallazgos en un paciente con cardiopatía coronaria}

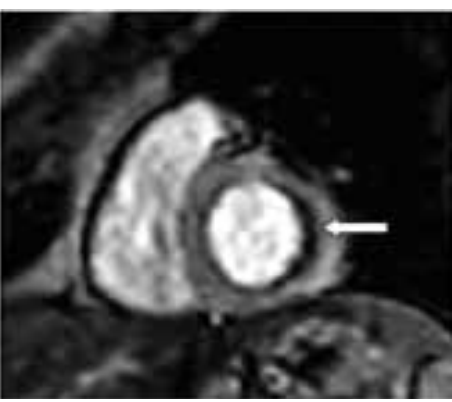

A

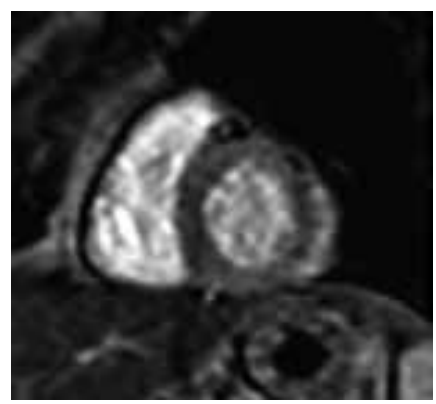

B

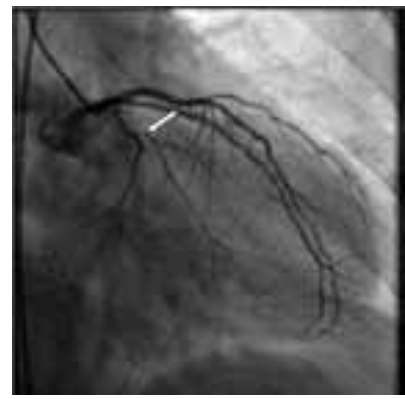

C

Figura 2. Resonancia y coronariografía de paciente con antecedente de angioplastía con stent en arteria lateral alta (marginal obtusa) y que ingresa por dolor torácico. En A se observa durante la fase de stress, falta de captación (flecha blanca) en cara lateral del ventrículo izquierdo. Durante la fase de reposo, B, se observa una recuperación de la perfusión en la misma zona, lo cual es compatible con isquemia. El estudio coronariográfico $(C)$ demostró lesión crítica en rama lateral de arteria circunfleja (flecha), tras lo cual se procedió a nueva angioplastía. 


\section{Discusión:}

En la práctica cardiológica habitual están disponibles distintos exámenes capaces de evaluar la presencia de isquemia miocárdica. De lo anterior se desprende que más que un nuevo examen lo que necesitamos los clínicos es tener test de isquemia no invasivos que sean certeros, reproducibles, sin uso de medios tóxicos y que sean costo/efectivos. El uso de la RMCPS es altamente atractivo en el escenario cardiológico actual dado las ventajas inherentes a la técnica. Como lo demuestra nuestra experiencia el uso de esta técnica no sólo es factible, sino también es segura, y posee una muy buena correlación con los hallazgos coronariográficos. En nuestra serie obtuvimos índices kappa altos tanto para el diagnóstico de enfermedad coronaria, como para la localización de la zona isquémica y su correlación con la anatomía coronaria. Información disponible en la literatura reportan índices de similares a los reportados en el presente trabajo ${ }^{5}$.

Una de las limitaciones de esta serie es que no se trata de un estudio ciego randomizado, sino de un registro clínico. Sin embargo, a pesar de esta limitación son pacientes que se ven a diario en la práctica clínica y no un grupo súperseleccionado, por lo cual los hallazgos serían extrapolables al resto de los pacientes. Además, en todos los casos de una RMCPS el estudio invasivo fue realizado e informado con posterioridad, lo cual le otorga una validez al informe del estudio no invasivo. El hecho que la decisión de coronariografía se haya basado en criterios clínicos podría ser un factor confundente, pero el análisis comparativo no encontró diferencias significativas entre los pacientes que se sometieron a RMCPS versus los que no.

En nuestro reporte aquellos pacientes que no presentaron una RMCPS positiva para isquemia, no tuvieron eventos clínicos adversos, lo cual el conferiría un valor predictivo en poblaciones como la reportada. Esto también ha sido publicado por otros autores en diversas experiencias, lo que cobra importancia cuando confrontamos la información provista por exámenes anatómicos (como la coronariografía o la tomografía computada de arterias coronarias) o cuando queremos evaluar función por sobre información anatómica, como puede ser el caso de poblaciones con alta prevalencia de enfermedad coronaria en los cuales interesa más la repercusión de ésta en la estratificación de riesgo ${ }^{6}$.

Futuros estudios de nuestro grupo consideran la realización de una experiencia randomizada y doble ciega, con el fin de consolidar este test no invasivo como un aporte a la práctica cardiológica. En este sentido la RMCPS tiene la ventaja de ser no invasiva, no ionizante y al tener además la capacidad de dar información complementaria (como anatomía cardíaca, función ventricular, viabilidad miocárdica) podría tener una mejor relación costo beneficio al poder prescindir de otros exámenes complementarios.

En suma, la RMCPS es una técnica factible, segura y que otorga una alta certeza diagnóstica en la detección de isquemia en pacientes con sospecha de cardiopatía coronaria.

\section{Referencias:}

1. ENBERGS A, BÜRGER R, REINECKE H, BORGGREFE M, BREITHARDT G, KERBER S. Prevalence of Coronary artery disease in a general population without suspicion of coronary artery disease: angiographic analysis of subjects aged 40 to 70 years referred for catheter ablation therapy. Eur Heart J. 2000; 21: 45-52.

2. CORSI C, LANG RM, VERONESI F, WEINERT L, CAIANI EG, MACENEANEY $P$, et al. Volumetric quantification of global and regional left ventricular function from real-time three-dimensional echocardiographic images. Circulation 2005; 112: 1161-70

3. KIM RJ, WU E, RAFAEL A, CHEN EL, PARKER MA, SIMONETTI O, et al. The use of contrast-enhanced magnetic resonance imaging to identify reversible myocardial dysfunction.

N Engl J Med 2000; 343: 1488-1490.

4. DOESCH C, SEEGER A, DOERING J, HERDEG C, BURGSTAHLER C, CLAUSSEN C, et al. Risk Stratification by Adenosine Stress Cardiac Magnetic Resonance in Patients With Coronary Artery Stenoses of Intermediate Angiographic Severity. J Am Coll Cardiol Img, 2009; 2: 424-433.

5. SAKUMA H, SUZAWA N, ICHIKAWA Y, MAKINO K, HIRANO T, KITAGAWA K, et al. Diagnostic accuracy of stress first-pass contrast-enhanced myocardial perfusion MRI compared with stress myocardial perfusion scintigraphy. AJR Am J Roentgenol. 2005; 185: 95-102.

6. PILZ G, JESKE A, KLOS M, ALI E, HOEFLING B, BERNHARDT P. Prognostic value of normal adenosine stress cardiac magnetic resonance imaging exams - one year follow-up results of a prospective study. Journal of Cardiovascular Magnetic Resonance 2008; 10: A22. 